Classification

Physics Abstracts

$62.65-61.40 \mathrm{D}$

\title{
Nonlinear relaxational ultrasonic absorption in metallic glasses
}

\author{
Yu. M. Galperin, V. L. Gurevich
}

A. F. Ioffe Institute for Technical Physics, Academy of Sciences of the U.S.S.R., Leningrad, U.S.S.R. and D. A. Parshin

M. I. Kalinin Polytechnical Institute, Leningrad, U.S.S.R.

(Reçu le 2 février 1984, révisé le 4 juin, accepté le 12 juin 1984)

\begin{abstract}
Résumé. - Nous avons développé une théorie de l'absorption ultrasonore de relaxation dépendant de l'intensité dans des métaux amorphes (des verres métalliques). L'absorption de relaxation est due à la modulation de la séparation entre niveaux dans les systèmes à deux niveaux par la déformation créée par l'onde acoustique.

La non-linéarité de la constante d'absorption se manifeste essentiellement quand l'amplitude de la modulation des niveaux excède $k T$.

Abstract. - We have developed a theory of intensity-dependent ultrasonic relaxational absorption in amorphous metals (metallic glasses). The relaxational absorption is due to the modulation of the interlevel spacing of the two-level systems by the periodic strain field of acoustic wave.

The nonlinearity of the absorption coefficient appears to be essential if the amplitude of the modulation of the interlevel spacing exceeds $k T$.
\end{abstract}

The purpose of the present paper is to discuss a new type of intensity dependent low temperature acoustic absorption in amorphous metals. In contrast to the well-known nonlinear resonant absorption (see [1]), this new type of intensity-dependent absorption may be called nonresonant, or relaxational. We believe that the absorption of this type has been recently observed by Hikata, Cibuzar and Elbaum [2] (see also [3-5]).

It is well known that acoustic properties of amorphous metals, as well as a lot of other lowtemperature properties, are determined by the two-level systems (TLS) [6]. The resonant absorption is due to the interaction of the acoustic wave with the TLS with the energy (interlevel spacing) $E=\hbar \omega$ where $\omega$ is the ultrasonic frequency. It becomes intensity-dependent if the sound intensity is so high that it shifts essentially the occupancies of the resonant TLS from their equilibrium values. The explicit form of the intensity dependence of the coefficient of resonant absorption is given by (1) :

$$
\Gamma^{(\mathrm{res})}=\frac{\pi N \gamma^{2}}{\rho v^{3}} \omega \tanh \frac{\hbar \omega}{2 k T} \frac{1}{\left(1+J / J_{\mathrm{c} 1}\right)^{1 / 2}}
$$

where $N$ is the density of states, $\gamma$ is the deformation potential constant describing the TLSstrain interaction, $\rho$ is the density of the amorphous metal, $v$ is the sound velocity, $J$ is the sound 
intensity while $J_{\mathrm{c} 1}$ is its critical value, and $T$ is the temperature. This expression adequately describes the contribution of those TLS whose relaxation times $\tau$ satisfy the inequality (see [1]).

$$
\omega \tau \gg 1+J / J_{\mathrm{c} 1} \text {. }
$$

The intensity-dependent absorption described by equation (1) was observed in a metallic glass by Doussineau et al. [7].

The criterion for the intensity dependence of the nonresonant absorption is quite different. The source of this dependence is periodic time variation of the energy $E$ (with the sound wave period $2 \pi / \omega)\left({ }^{1}\right)$. Nonlinear behaviour becomes essential if the amplitude of the energy variation, $d$, is of the order of or bigger than $k T$. For a better understanding of our physical arguments let us begin with the discussion of strong nonlinearity, $d \gg k T$ (though on the experiments [2-5] this inequality has not been too strong).

If $d \gg k T$, the TLS can be excited only during a small part of the period. Its relative duration, $k T / d$, being intensity-dependent, the whole kinetics of the TLS is essentially determined by the sound intensity.

On the other hand, the relaxation time $\tau$ depends strongly on $E$. Therefore the relaxation of the excited TLS and the energy emitted by them during the relaxation process is determined by the form of this dependence at energies $E \gtrsim k T$.

The nonlinear absorption observed in [3-5] had the same intensity dependence as (1) but the coefficient at $\left(1+J / J_{\mathrm{c}}\right)^{-1 / 2}$ was a linear function of $\omega$ and was almost independent of $T$. This dependence differs from the one predicted by equation (1) where at $\hbar \omega \ll k T, \Gamma^{\text {(res) }}(0) \sim \omega^{2} / T$. Note that the frequency and temperature intervals in [7] and [3-5] partially overlapped.

In a recent paper [2] it has been pointed out that the results of papers [7] and [3-5] were obtained at different sound intensities $J$. The sound intensity increasing, we at first come to the region 1 where the intensity-dependent part of $\Gamma(J)$ is described by equation (1), and then to region 2 where the absorption behaves according to [3-5].

We believe that at $J \gg J_{\mathrm{c} 1}$ the resonant contribution to the absorption coefficient saturates and becomes negligibly small as compared to another contribution to the absorption which saturates at much higher intensities. The latter may be called relaxational, or nonresonant. In the linear regime it has been investigated by Jäckle [9] for dielectric glasses and by Black and Fulde for amorphous metals $[6,10]$.

The nonresonant absorption is due to the modulation of the energy $E\left({ }^{2}\right)$ of the TLS by the periodic strain field of acoustic wave. As a result, the level population is displaced from its equilibrium value for it takes a finite time $\tau$ to restore the equilibrium while the energy $E$ changes continuously. Such a shift results in energy dissipation and thus in sound attenuation.

We shall assume (see Black [6]) that the relaxation time is determined by the interaction of the TLS with the equilibrium conduction electrons. $\tau$ depends not only on the energy $E$ but also on the tunnel parameter $\Delta_{0}$ and temperature $T$. In the first approximation of the perturbation theory we have [6]

Where

$$
\frac{1}{\tau}=\frac{\pi}{4 \hbar} \chi \frac{\Delta_{0}^{2}}{E} \operatorname{coth} \frac{E}{2 k T}
$$

$$
\chi=\frac{\overline{V^{2}}}{V_{0}^{2}}
$$

( $\left.{ }^{1}\right)$ The intensity-dependent nonresonant absorption has also been considered by Arnold et al. [8]. They have used an entirely different physical approach. A comparison of the two approaches will be made below.

$\left({ }^{2}\right)$ One can consider $E$ as a function of time $t$ only in the adiabatic approximation. We suppose that the frequency $\omega$ is sufficiently small, so that the approximation is applicable (see Eq. (13) below). 
is a dimensionless parameter of TLS-electron coupling ; $\overline{V^{2}}$ is an average value of the corresponding matrix element squared, the average being taken over different electron states, while $1 / V_{0}$ is the electron density of states at the Fermi level, per one electron.

Because of the relation $E=\left(\Delta^{2}+\Delta_{0}^{2}\right)^{1 / 2}$ where $\Delta$ is the asymmetry of the effective potential of the TLS, $\Delta_{0}$ cannot be bigger than $E$. Thus, for a given $E$, a minimum value of $\tau$ exists which is given by

$$
\frac{1}{\tau_{\min }(E)}=\frac{\pi \chi}{4 \hbar} E \operatorname{coth} \frac{E}{2 k T}
$$

If $E \ll k T$ we have

$$
\frac{1}{\tau_{\min }}=\frac{\pi}{2} \chi \frac{k T}{\hbar} \equiv \frac{1}{\tau_{0}}
$$

i.e. $\tau_{\min }$ is independent of $E$.

Under the influence of periodic deformation the energy $E$ varies with time as (see Fig. 1)

$$
E(t)=\sqrt{\Delta_{0}^{2}+(\Delta+d \cos \omega t)^{2}} .
$$

Where $d$ is the amplitude of the modulation of the asymmetry $\Delta_{0}$ due to the acoustic wave; $d=2 \gamma_{\mathrm{i} 1} u_{\mathrm{i} 1}^{(0)} ; u_{\mathrm{i} 1}^{(0)}$ is the strain amplitude.

The rigorous theory should be based on the solution of the balance equation using this form of time dependence of the energy $E(t)$ and subsequent summation over all the TLS that can take part in the absorption. This theory will be published elsewhere [11].

However to make most of the order-of-magnitude estimates for the extreme nonlinear case $d \gg k T$ one needs only relatively simple physical considerations $\left({ }^{3}\right)$.



Fig. 1. - Time dependence of the interlevel spacing of a two-level system produced by the periodic variation of the strain in the acoustic wave. The active regions are shaded.

$\left({ }^{3}\right)$ Such considerations have been used by the authors in [12]. However, we have not considered in [12] an important physical phenomenon of the protraction of relaxation which will be discussed below in this paper. 
Under the condition $d \gg k T$ the whole period of acoustic field variation, $2 \pi / \omega$, can be divided into active and passive regions. The active regions are determined by the inequality $E \lesssim k T$ while the passive regions constitute the remaining part of the period. In the active regions the TLS can emit as well as absorb energy. In the passive regions the TLS can only emit energy exciting the electrons.

By definition, the active regions exist only for the TLS with $\Delta_{0} \lesssim k T, \Delta \lesssim d$. If $\Delta>d$, or $\Delta_{0}>k T$ the interlevel spacing $E$ remains bigger than $k T$ during the whole period; such TLS do not contribute the absorption.

The width of an active region is of the order of

$$
t_{\mathrm{a}}=\frac{k T}{d} \frac{1}{\omega} .
$$

Thus we naturally come to the two limiting cases

and

$$
\text { 1) } t_{\mathrm{a}} \ll \tau_{0}, \text { or } \omega \tau_{0} \gg k T / d
$$

$$
\text { 2) } t_{\mathrm{a}} \gg \tau_{0}, \text { or } \omega \tau_{0} \ll k T / d \text {. }
$$

The first case is simpler for consideration. In this case the probability of excitation of a TLS during its passage through an active region is small : it is of the order of $t_{\mathrm{a}} / \tau$. In other words, it takes many periods to excite such a TLS. The smaller is $\tau$, the bigger is the probability, so the main contribution to the absorption comes from the TLS with $\Delta_{0} \simeq k T$, which have $\tau \simeq \tau_{0}$ in the whole active region $\left({ }^{4}\right)$.

If such a TLS has been excited in the active region it will remain excited during roughly the same number of periods as above. This is a consequence of the linear dependence of $\tau\left(E, \Delta_{0}\right)$ on $E$ at $E>k T$. This number is the inverse probability $\tau_{0} / t_{\mathrm{a}}$. Thus the average time when the TLS is the excited state is of the order of $\tau_{0} / \omega t_{\mathrm{a}}$ which is equal to $\tau\left(E, \Delta_{0}\right)$ at $E \simeq d$ and $\Delta_{0} \simeq k T$. So the order of magnitude of the energy emitted by such a TLS is $d$. Thus the average power transferred by the TLS to the conduction electron system may be estimated as

$$
\frac{t_{\mathrm{a}} \omega d}{\tau_{0}}=\frac{k T}{\tau_{0}} .
$$

To get the total power density $\boldsymbol{P}$ absorbed in the amorphous metal we multiply this quantity by the total number $N d$ of the TLS $\left(\right.$ per $\mathrm{cm}^{3}$ ) that can take part in the absorption $\left({ }^{5}\right)$. Thus

$$
P \simeq \frac{N k T d}{\tau_{0}(T)}=P_{0}\left(\frac{J_{\mathrm{c} 2}}{J}\right)^{1 / 2} ; J \gg J_{\mathrm{c} 2} .
$$

Where $P_{0} \simeq N d^{2} / \tau_{0}(T)$ is the absorbed power in the linear regime while $J_{c 2}$ is the critical sound intensity for the nonresonant absorption. We shall define $J_{\mathrm{c} 2}$ by the equation $d=k T$, or

$$
J_{\mathrm{c} 2}=\frac{1}{2} \rho v^{3}\left(\frac{k T}{2 \gamma}\right)^{2}
$$

${ }^{(4)}$ Of course a TLS with $\Delta_{0} \ll k T$ has a relaxation time of the order of $\tau_{0}$ at the moments when its energy $E(t)$ is of the order of $\Delta_{0}$. However, as one can easily check,-the contribution of these TLS is relatively small because of their rapid passage through the energy interval of the order of $\Delta_{0}$.

$\left({ }^{5}\right)$ Note that the total number of TLS with $\Delta_{0} \lesssim k T$ because of distribution $N\left(\Delta, \Delta_{0}\right) \sim 1 / \Delta_{0}$ can have only logarithmic dependence on temperature $T$. 
where $v$ is the sound velocity, and $\gamma$ is the deformation potential constant describing the TLSstrain interaction.

To get the absorption coefficient $\Gamma$ one should divide (9) by the acoustic flux density.

The second case is much more complicated for the dependence $\tau\left(\Delta_{0}\right)$ is to be considered in detail. However, in our opinion, this is the case which directly relates to the experiment discussed in [2-5]. Let us divide all the TLS that can contribute to the absorption into two kinds. The first kind is characterized by small values of $\tau$ :

$$
\int_{\mathrm{a}} \frac{\mathrm{d} t}{\tau}>1
$$

where the integration is performed over an active region. This inequality means that all the processes that can happen with these TLS take place in the active regions. In other words, in these regions the relaxation is rapid. Therefore the characteristic energy transferred to the electron system per one period by such a « rapid " TLS is of the order of $k T$. The number of TLS that can contribute to the absorption is, as above, of the order of $N d$. As a result, we get the following estimate for the contribution of the « rapid » TLS to the density of the absorbed power

$$
P^{(\mathrm{r})} \simeq N \omega k T d
$$

There is another kind of TLS that may be called « slow » for which

$$
\int_{\mathrm{a}} \frac{\mathrm{d} t}{\tau}<1 .
$$

In dielectric glasses their contribution would be of the same order as that of the " rapid » TLS [13]. In amorphous metals, however, it has different intensity and frequency dependence. The " slow " TLS are responsible for an interesting physical phenomenon typical for metallic glasses that may be called protraction of relaxation.

It originates in the fact that $\tau$ is an increasing function of energy $E$ (provided $E>k T$ ). This is why it takes the time of the order of the whole period to deexcite the TLS excited during a small (active) part of the period. As a result, the energy transferred to the electron system by an excited TLS is of the order of $d$. These considerations give the contribution of the " slow " TLS to the absorbed power of the order of the linear one

$$
P_{0} \simeq N d^{2} \omega
$$

A more rigorous quantitative calculation based on the solution of the balance equation gives additional logarithmic dependence on the intensity of the form (see [11])

$$
P^{(s)} \simeq P_{0} / \log \frac{d}{k T \omega \tau_{0}(T)}
$$

We see that in the second case the total nonlinear absorption coefficient $\Gamma$ is a sum of two terms. The first one decreases appreciably with the intensity $J\left(\right.$ as $\left.J^{-1 / 2}\right)$; the second one is almost intensity-independent.

We believe that our theory (in the second case $t_{\mathrm{a}} \gg \tau_{0}$ ) can explain the two-step intensity dependence of the absorption coefficient observed in [2]. The first step reflects the intensity dependence of the resonant absorption while the second step is related to the intensity dependence of the nonresonant absorption by the « rapid » TLS. The critical intensity $J_{\mathrm{c} 2}$ is proportional to $T^{2}$ which also coincides with the experimental data [3-5]. It is proportional to $v^{3}$ which qualitati- 
vely agrees with the fact that the nonlinear behaviour is much more pronounced for transverse than for longitudinal sound.

Let us give estimates for the critical intensity $J_{\mathrm{c} 2}$ (characterizing the intensity dependence for both cases, 1 and 2, considered above). For the values of the parameters $T=0.3 \mathrm{~K}, \rho=10 \mathrm{~g} / \mathrm{cm}^{3}$, $v=1.8 \times 10^{5} \mathrm{~cm} / \mathrm{s}, \gamma=2 \mathrm{eV}$ we get from equation (10) $J_{\mathrm{c} 2}=0.1 \mathrm{~W} / \mathrm{cm}^{2}$. It is also instructive to compare the values which the theory gives for the critical intensities $J_{\mathrm{c} 1}$ and $J_{\mathrm{c} 2}$. Employing equations $(5,10)$ we have $J_{\mathrm{c} 2} / J_{\mathrm{c} 1} \sim \chi^{-2}$. If, for instance, $\chi \simeq 0.3$ then $J_{\mathrm{c} 2} / J_{\mathrm{c} 1} \simeq 10^{3}$.

Arnold, Doussineau and Levelut in their paper [8] have also suggested that the relaxational absorption in amorphous metals can decrease with intensity. In such a way they wished to explain the experimental data [2-5]. To calculate the change of the distribution function of the thermal TLS under the influence of the acoustic wave of the frequency $\omega$ they have used the resonant approximation. We think that it is justified if the condition $\hbar \omega \simeq E$ is satisfied with sufficient accuracy (see (2) and [1] where the derivation of the resonant absorption is given along with the discussion of the conditions of its applicability). However, it is more difficult to justify it for the case $\hbar \omega \ll E$. Here $E$ is the typical value of the TLS energy, of the order of $k T$.

We believe that the adiabatic pprovimation we have used in our calculation is more appropriate for the case of sufficiently acoustic frequencies. One can show that the conditions of its applicability are [13]

$$
\hbar \omega \ll E, \quad \hbar|\mathrm{d} E / \mathrm{d} t| \ll E^{2} .
$$

They are satisfied for energies of the order of $d$ or $k T$.

It is worthwhile to mention that, within this approximation, diagonalization of both the Hamiltonian and the density matrix is possible. In other words, both types of interaction of a TLS with strain are taken into account, i.e. the diagonal $D$-term, and the off-diagonal $M$-term (see $[1,6]$ for their definition). This is an additional argument why, as we think, it is pointless in this case to separate the resonant contribution from the total absorption.

The principal points where the predictions of our theories differ are :

1) we predict in the extreme nonlinear regime existence of two frequency intervals (7) and (8), the transition from the first to the second one being determined by the intensity-dependent parameter $\omega \tau_{0}(T) d / k T$. 2) In the low-frequency interval our theory gives the contributions proportional to

$$
1 / \log \frac{d}{k T \omega \tau_{0}(T)}
$$

i.e. almost independent of the intensity.

Finally we wish to discuss the following point. The nonlinear nonresonant absorption is extremely sensitive to the energy dependence of $\tau$. The whole picture of intensity dependence of the nonresonant absorption may be especially interesting for superconducting amorphous metals where different relaxation mechanisms can compete. Indeed, the number of normal excitations in a superconductor decreases exponentially with decreasing temperature. According to Black and Fulde [10], the inverse relaxation time of the TLS with $E<2 \Delta_{\mathrm{s}}$ where $2 \Delta_{\mathrm{s}}$ is the superconducting gap, should behave in the same way. On the contrary, the relaxation time of the TLS with $E>2 \Delta_{\mathrm{s}}$ is of the order of that for the normal state because these high energy TLS can relax via breaking the Cooper pairs. Thus the TLS excited in the active region remains in the excited state until the splitting of its levels exceeds the superconducting gap.

Another interesting point is a possible competition of the two mechanisms of relaxation in superconducting amorphous metals. Ifthe number of normal excitations is reduced the phonon mechanism may become important.

Thus investigation of intensity-dependent nonresonant absorption may prove to be a very sensitive tool for the study of various aspects of TLS-electron and TLS-phonon interaction in amorphous metal, both in the normal and in the superconducting states. 
Finally, it might be worthwhile to mention that the same theoretical methods can be employed to investigate the intensity dependence of the nonresonant contribution to the sound velocity in amorphous metals.

\section{References}

[1] Hunklinger, S., Arnold, W., in Physical Acoustics, Ed. by W. P. Mason, R. N. Thurston (Academic N.Y.) 1976, 12, 155.

[2] Hikata, A., Cibuzar, G., Elbaum, C., J. Low Temp. Phys. 49 (1982) 339.

[3] Araki, H., Park, G., Hikata, A., Elbaum, C., Phys. Rev. B 21 (1980) 4470.

[4] Park, G., Hikata, A., Elbaum, C., Phys. Rev. B 24 (1981) 7389.

[5] Park, G., Hikata, A., Elbaum, C., J. Non Cryst. Solids 45 (1981) 93.

[6] BlaCK, J. L., in Metallic Glasses, edited by H. J. Güntherodt (Springer-Verlag, N.Y.) 1980, p. 167.

[7] Doussineau. P., Legros. P., Levelut, A., Robin, A.. J. Physique Lett. 39 (1978) L-265.

Doussineau, P., J. Physique Lett. 42 (1981) L-83.

[8] Arnold, W., Doussineau, P., Levelut, A., J. Physique Lett. 43 (1982) L-695.

[9] JÄCKLE, J., Z. Phys. 257 (1972) 212.

[10] Black, J. L., Fulde, P., Phys. Rev. Lett. 43 (1979) 453.

[11] Gal'Perin, Yu. M., Gurevich, V. L., Parshin, D. A., JetP 86 (1984) 1900.

[12] Gal'perin, Yu. M., Gurevich, V. L., Parshin, D. A., Pis'ma Zh. Eksp. Teor. Fiz. 36 (1982) 386 (JETP Lett. 36 (1982) 466).

[13] GAL'PERIN, Yu. M., Zh. Eksp. Teor. Fiz. 85 (1983) 1386. 